\title{
High-Throughput Selection of Effective RNAi Probes for Gene Silencing
}

\author{
Rajeev Kumar, Douglas S. Conklin, and Vivek Mittal ${ }^{1}$ \\ Cancer Genome Research Center, Cold Spring Harbor Laboratory, Woodbury, New York 11797, USA
}

\begin{abstract}
RNA interference (RNAi) is a process of sequence-specific posttranscriptional gene silencing mediated by double-stranded RNA. RNAi has recently emerged as a powerful genetic tool to analyze gene function in mammalian cells. The power of this method is limited however, by the uncertainty in predicting the efficacy of small interfering RNAs (siRNAs) in silencing a gene. This has imposed serious limitations not only for small-scale but also for high-throughput RNAi screening initiatives in mammalian systems. We have developed a reliable and quantitative approach for the rapid and efficient identification of the most effective siRNA against any gene. The efficacy of siRNA sequences is monitored by their ability to reduce the expression of cognate target-reporter fusions with easily quantified readouts. Finally, using microarray-based cell transfections, we demonstrate an unlimited potential of this approach in high-throughput screens for identifying effective siRNA probes for silencing genes in mammalian systems. This approach is likely to have implications in the use of RNAi as a reverse genetic tool for analyzing mammalian gene function on a genome-wide scale.
\end{abstract}

[Supplemental material is available online at www.genome.org. The following individuals kindly provided reagents, samples, or unpublished information as indicated in the paper: G. Hannon, J. Silva, R. Benezra, K. LaVine, S. Gupta, D. Helfman, E. Hatchwell, S. Kantaraci, and A. Lassar.]

RNA interference (RNAi) is a process by which double-stranded RNA (dsRNA) homologous to the target locus can specifically inactivate gene function in plants, invertebrates, and mammalian systems (Sharp 1999; Hammond et al. 2001). The endogenous mediators of sequence-specific mRNA degradation are 21and 22-nucleotide (nt) siRNAs generated from longer dsRNAs by the ribonuclease III activity of the evolutionarily conserved dicer enzyme (Bernstein et al. 2001; Elbashir et al. 2001b). Genespecific long dsRNAs have been successfully used in Caenorhabditis elegans and Drosophila melanogaster for RNAi-mediated gene silencing (Hannon 2002). In mammalian cells, however, dsRNAs longer then 30 base pairs (bp) trigger the antiviral/interferon pathways, which results in global shut-down of protein synthesis (Marcus and Sekellick 1985; Gil and Esteban 2000). It was recently demonstrated that RNAi-mediated gene silencing can be obtained in cultured mammalian cells by delivery of either chemically synthesized short $(<30-n t)$ double-stranded siRNA molecules (Elbashir et al. 2001a) or by endogenous expression of short hairpin RNAs (shRNAs) bearing fold-back stem-loop structure (Brummelkamp et al. 2002; Elbashir et al. 2002; Paddison et al. 2002; Paul et al. 2002; Sui et al. 2002).

General guidelines for designing siRNA oligonucleotides are available (Elbashir et al. 2002) which require avoiding regions of mRNA such as 5' and 3' untranslated regions (UTRs) and regions within 75 bases of a start codon, and sequences having a GC content of $>70 \%$ or $<30 \%$. Although the existing rules for siRNA selection are a reliable general guide, they do not ensure that each selected siRNA will work. For example, even though targeting the UTR is not generally recommended, successful gene inhibition has been reported for siRNAs targeting 3' UTRs of the Myc oncogene (Bryom et al. 2002) and CD8 $\alpha$ receptor (McManus

\section{'Corresponding author.}

E-MAIL mittal@cshl.org; FAX (516) 422-4109.

Article and publication are at http://www.genome.org/cgi/doi/10.1101/ gr.1575003. et al. 2002). Thus it has been observed that in many cases a majority of siRNAs or shRNAs designed against a gene are not effective (Elbashir et al. 2001a; Holen et al. 2002; Lee et al. 2002; Yu et al. 2002; Kapadia et al. 2003). On average, only 1 in 5 of the siRNAs/shRNAs selected for targeting a region of a gene show efficient gene silencing (McManus et al. 2002; Kapadia et al. 2003; R. Kumar and V. Mittal, unpubl.). Instability of an siRNA probe in vivo, inability to interact with components of the RNAi machinery, and inaccessibility of the target mRNA due to local secondary structural constraints have all been proposed as possible causes for the failure of most tested siRNAs. An analysis of nucleotide sequences, melting temperatures, and secondary structures has thus far not revealed any obvious differences between effective and noneffective siRNA (Hohjoh 2002), thus preventing the development of more effective siRNA design methods. Moreover, empirical approaches such as RNAse H susceptibility assays of siRNA/target duplexes (Lee et al. 2002) and shotgun approaches in which a mixture of siRNA produced by RNAse III-mediated hydrolysis of long double-stranded RNA is used (Calegari et al. 2002; Yang et al. 2002) do not easily translate to the design of effective RNAi probes. At present, the lack of a reliable target-site selection method has imposed serious limitations on the efficiency of RNAi as a general tool for gene function analysis.

We have developed a quantitative procedure for rapid identification of an effective siRNA/shRNA for inhibition of target gene expression. Effective RNAi probes are identified based on their ability to inactivate cognate sequences in an ectopically expressed target gene-reporter chimeric mRNA. Using either a fluorescent or enzymatic reporter, the siRNA effect is monitored quantitatively. By examining a variety of genes with diverse biological functions in various mammalian cell lines, we have shown a strong correlation in the suppression of ectopically expressed and cognate endogenous genes by siRNA probes. Furthermore, using microarray-based cell transfections, we demonstrate that this approach can be tailored to high-throughput screens for identifying effective siRNA probes in mammalian systems. Such 
a screen would have unlimited potential for analyzing mammalian gene function on a genome-wide scale.

\section{RESULTS AND DISCUSSION}

\section{Experimental Design for Selecting Effective} siRNA Probes

The crucial event in RNAi-mediated gene silencing is the interaction of a 21-nt siRNA probe with its complementary sequence within an mRNA. This led us to hypothesize that the expression of an endogenous gene will be as efficiently silenced as that of

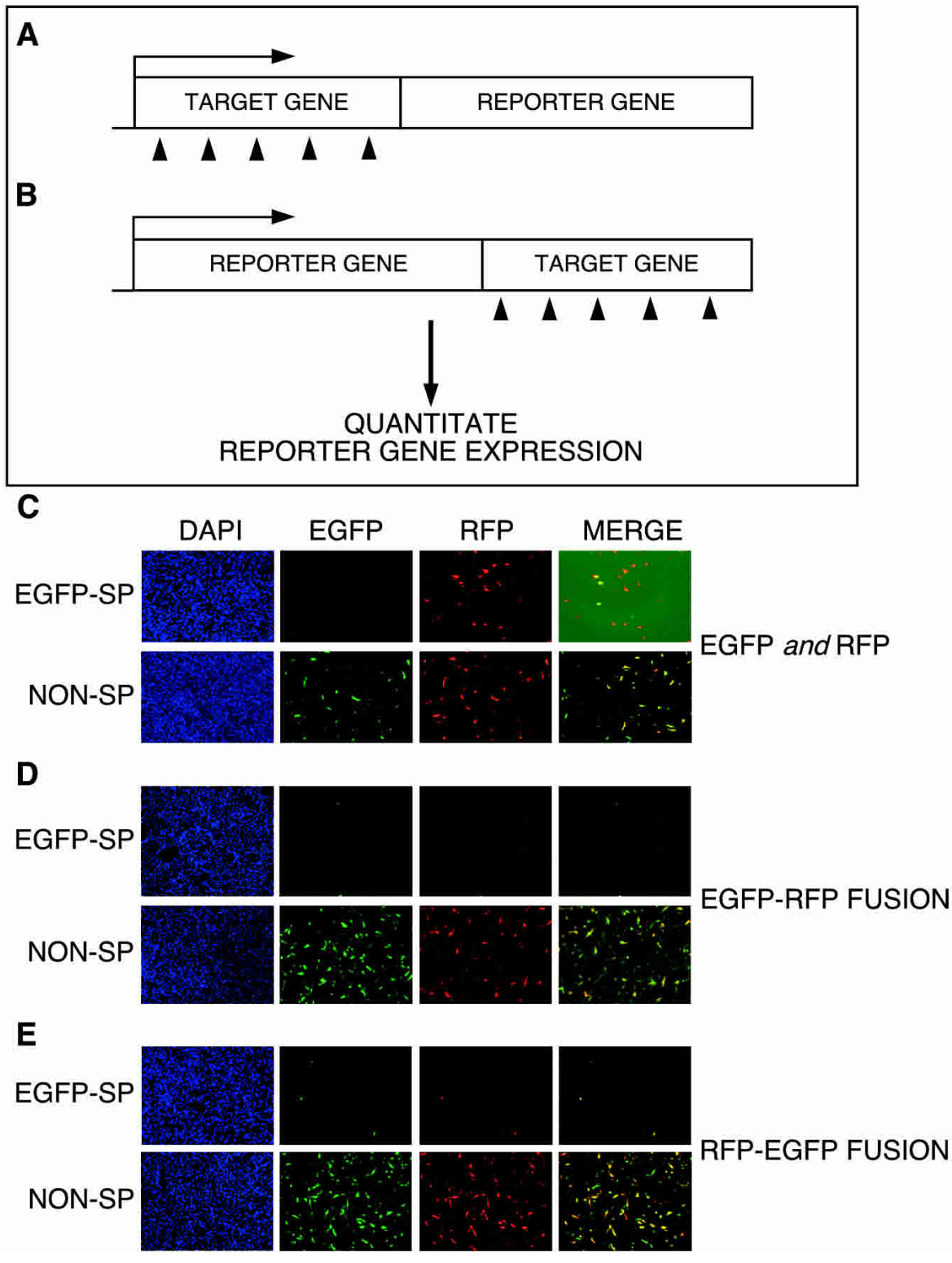

Figure 1 Strategy and experimental verification for screening effective siRNA probes using targetreporter fusion. $(A)$ Reporter gene fused at either C-terminal or $(B) \mathrm{N}$-terminal of target gene. A panel of siRNAs or shRNAs against target gene is shown $(\mathbf{\Lambda})$. Efficacy of siRNA-mediated target gene silencing was measured by quantitation of reporter gene expression. $\mathrm{C} 2 \mathrm{C} 12$ cells were cotransfected with either EGFP-specific siRNA (upper panel) or nonspecific siRNA (lower panel) with (C) two independent plasmids expressing EGFP and RFP, $(D)$ a plasmid expressing EGFP-RFP fusion protein, and $(E)$ a plasmid expressing RFP-EGFP fusion protein. DAPI, 4', 6'-diamidino-2-phenylindole hydrochloride; EGFP, enhanced green fluorescent protein; RFP, red fluorescent protein; NON-SP, nonspecific siRNA; SP, specific siRNA. A scrambled siRNA served as a nonspecific control. ectopically expressed mRNA by an effective siRNA. We designed constructs that express target protein fused at either the $\mathrm{N}$ - or $\mathrm{C}$-terminus to a fluorescent (such as enhanced green fluorescent protein, EGFP or red fluorescent protein, RFP) or enzymatic (Renilla luciferase) reporter polypeptide (Fig. 1). These constructs were transfected into mammalian cells together with individua siRNAs designed against various regions of the target gene (Fig. $1 \mathrm{~A}, \mathrm{~B})$. If any siRNA effectively targets and inactivates expression of its cognate mRNA, a marked reduction in reporter expression (EGFP/RFP fluorescence or enzymatic activity) is expected. Conversely if it fails to efficiently target its cognate mRNA, no significant change in reporter expression should be observed. Both the fluorescence and enzymatic activities can be quantitated.

The feasibility of the experimental design was tested by evaluating critical parameters associated with the targetreporter fusion products such as stability of fusion proteins, accessibility of target site in the chimeric mRNA, and specificity of siRNA probes in suppressing cognate mRNA expression as reflected by changes in reporter expression. EGFP and RFP were used as a target and reporter respectively, and an effective siRNA specific to EGFP mRNA was used (Donze and Picard 2002). A plasmid expressing EGFP was cotransfected with an effective siRNA targeting specifically EGFP mRNA (Donze and Picard 2002) with a control plasmid expressing RFP. A significant reduction in EGFP, but not in RFP, expression was observed (Fig. 1C, upper panel), demonstrating specificity of the siRNA for the target gene. Transfection of the cells with a nonspecific (NON-SP) siRNA affected neither EGFP nor RFP expression (Fig. 1C, lower panel). We then tested both an $\mathrm{N}$ terminal and C-terminal target-reporter fusion (EGFP-RFP and RFP-EGFP) to determine whether siRNA against the target (EGFP) would result in the abrogation of reporter (RFP) gene expression. The results showed reduced expression of EGFP and RFP for both N- and C- terminal fusions (Fig. 1D,E, upper panel) as compared to nonspecific siRNA (Fig. $1 \mathrm{D}, \mathrm{E}$, lower panel), suggesting that the siRNA-mediated suppression of the target gene (EGFP) expression is faithfully reported by the reporter (RFP) portion of the fusion constructs. Taken together the data suggest that the target-reporter products are stable and that the reporter portion of the fusion construct accurately reflects silencing of the target when fused to either its $\mathrm{N}$ - or Cterminus. The latter property is particularly attractive, because it allows for flexibility in the construction of chimeric fusions.

To provide flexibility in the choice of reporter systems, we also explored the use of an enzymatic rather than a fluo-

\section{Genome Research \\ www.genome.org}


rescent reporter. Plasmids encoding an EGFP-Renilla luciferase fusion protein and internal control firefly luciferase, respectively, were transfected with varying concentrations of either EGFPspecific or nonspecific siRNAs. The internal control was used to normalize experimental variation associated with transfection efficiency. The results showed a specific dose-dependent decrease in EGFP expression with 50-250 ng of siRNA (Fig. 2A; upper panel). In contrast, nonspecific siRNA had no detectable effect on EGFP expression in this range (Fig. 2A, lower panel). Moreover, normalized quantitation of Renilla and firefly luciferase activity from lysates of aliquots of the same transfected cells showed that EGFP siRNA specifically decreases Renilla luciferase activity in a dose-dependent manner (Fig. 2B), consistent with the decrease in EGFP fluorescence (Fig. 2A). An approximately fivefold reduction in Renilla luciferase by specific siRNA relative to a control nonspecific siRNA was observed. Thus, both a fluorescent and an enzymatic reporter can be used effectively to monitor RNAimediated gene silencing. In alternative embodiments, a reporter gene which encodes a protein sequence such as His-tags, immunoglobulin domains, myc tags, poly-glycine tags, FLAG tags, or HA-tags may be used. These reporters can be conveniently detected by immunoassay methods, such as Western blotting, immunohistochemistry, ELISA, and/or immunoprecipitation.

\section{Application of the Method in Identifying Effective siRNA Probes}

To evaluate the usefulness of the screening method for identifying effective siRNA probes, we designed five siRNAs targeting various regions spanning the MyoD coding sequence (Fig. 3A). The $M y o D$ gene was used for the first screen because of its robust expression in muscle precursor cells and the availability of reliable antibodies to monitor levels of the protein. Cells were cotransfected with a construct expressing a MyoD-EGFP fusion protein, the dSRed2-N1 plasmid as an internal control for transfection, and individual siRNA probes. siRNA 25 showed dramatic reduction in the number of EGFP-positive cells compared to cells transfected with nonspecific siRNA (NON-SP, Fig. 3B), as determined by fluorescence microscopy. The normalized fluorescence intensity ratio of target (MyoD-EGFP) to internal control (RFP) was obtained from lysates of aliquots of the same transfected cells. The results show that siRNA 25 was the most effective (almost $50 \%$ reduction in GFP expression relative to nonspecific
siRNA) in the suppression of ectopic $M y o D$ gene expression (Fig. 3C), in agreement with the microscopic imaging (Fig. 3B). Partial reduction in normalized GFP fluorescence was observed with siRNA 294 (20\%) and siRNA 538 (10\%). To determine whether there is a correlation between the ability of the siRNA to inhibit expression of both ectopically expressed MyoD-EGFP and endogenous MyoD, cells were transfected with only siRNAs specific to MyoD. The level of endogenous MyoD was then examined by Western blot analysis. We observed a strong correlation between suppression of ectopic MyoD-EGFP and endogenous MyoD gene expression by the same panel of siRNAs (Fig. 3D, see percent reduction in MyoD expression), with siRNA 25 being the most effective (80\% reduction) in silencing endogenous MyoD expression (Fig. 3D, upper panel) followed by siRNA 294 (35\%) and siRNA 538 (10\%).

This strategy was then assayed for selecting effective plasmid-encoded shRNA. Plasmid-encoded shRNAs have distinct advantages over their chemically synthesized siRNA counterparts. They are cost-effective and provide a stable and continuous expression of hairpin RNA that is useful for analysis of phenotypes that develop over extended periods of time as a result of loss of gene function. We chose five different target sequences for MyoD hairpins, as shown in Figure 3E. shRNA 708 showed a dramatic reduction in the number of EGFP-positive cells (Fig. 3F, upper right panel) compared to nonspecific shRNA (Fig. 3F, upper left panel) or other shRNAs (data not shown). The normalized fluorescence intensity ratio of target (MyoD-EGFP) to internal control RFP was quantitated and confirmed the effectiveness of shRNA 708 (almost 65\% reduction in GFP expression relative to nonspecific siRNA), consistent with the microscopy imaging analysis (Fig. 3F). To monitor the ability of the various shRNAs to inhibit expression of the endogenous MyoD gene, cells were transfected only with shRNAs. Western blot experiments showed that shRNA 708 (85\% reduction in MyoD protein levels) was the most effective in suppressing endogenous MyoD gene expression (Fig. 3G, upper panel) followed by shRNA 312 and shRNA 897 (50\% reduction in each case), in agreement with the fluorescent intensity results (Fig. 3F).

We next determined the general applicability of the siRNA screening strategy in identifying effective RNAi candidates for additional genes. The silencing of Lamin A/C by a panel of siRNAs was analyzed (Fig. 4A). siRNA 608, which was previously shown to be effective against Lamin A/C (Harborth et al. 2001),
A

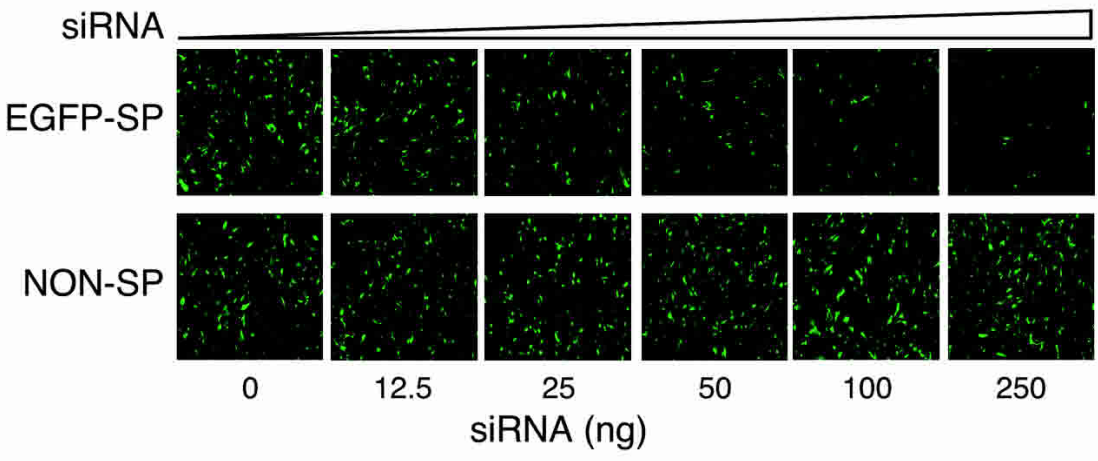

B

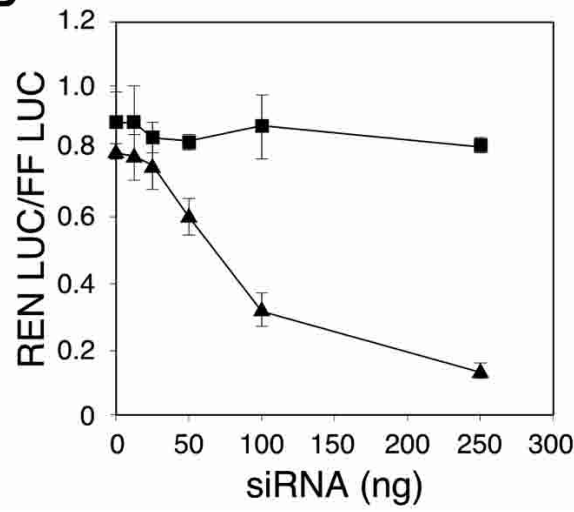

Figure 2 Quantitation of siRNA-mediated target gene silencing. ( $A$ ) C2C12 cells were transfected with a constant amount of EGFP-Rluc (expressing EGFP-Renilla luciferase fusion protein), pGL3-Control (expressing firefly luciferase as internal control), and increasing concentrations of EGFP-specific siRNA (upper panel) and nonspecific siRNA (lower panel). At $24 \mathrm{~h}$ posttransfection cells, were imaged for EGFP expression. (B) Normalized relative amount of Renilla and firefly luciferase $(n=3)$ as a function of treatment with nonspecific siRNA ( $\mathbf{\square})$ or EGFP-specific siRNA ( $\mathbf{\Delta})$ were plotted. Cotransfection without siRNA served as a control. A scrambled siRNA served as a nonspecific control. 
A $\begin{array}{lllll}25 & 294 & 438 & 538 & 637\end{array}$

B
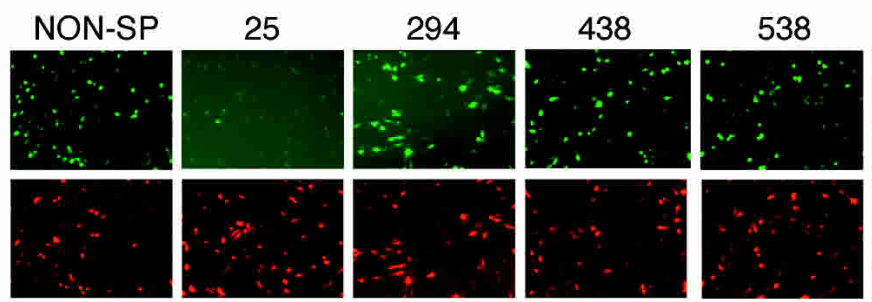

D

C
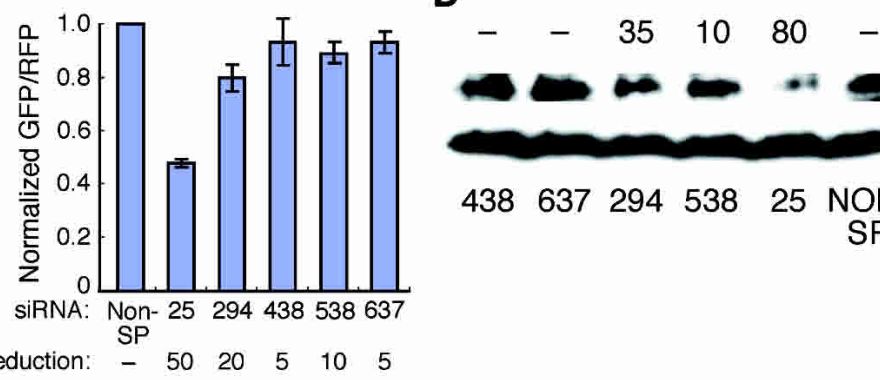

$\mathbf{E}$

1

312

507

708

$\mathbf{F}$

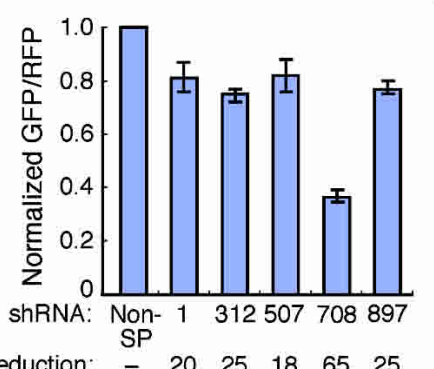

G
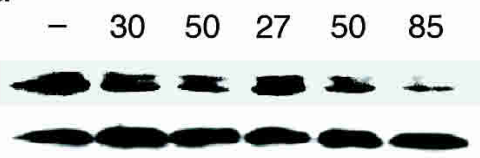

NON- $1 \quad 897507312708$

$\mathrm{SP}$

\% reduction: - $20 \quad 25 \quad 18 \quad 65 \quad 25$

Figure 3 Correlation between siRNA/shRNA screening results and suppression of endogenous MyoD. $(A)$ Schematic showing location of various siRNA probes against MyoD gene. The location of siRNA probes on the target sequences were assigned with respect to the translation start site (nucleotide position 1). (B) C2C12 cells were transfected with MyoD-EGFP, pDsRed2-N1 (internal control), and specific siRNAs against MyoD or a nonspecific siRNA as indicated. Upper and lower panels show EGFP and RFP expression, respectively, after $24 \mathrm{~h}$ of transfection. (C) Normalized relative amounts of EGFP and RFP for each siRNA. Percentage (\%) reduction in GFP fluorescence is indicated below each siRNA $(D)$ C2C12 cells were transfected with specific MyoD siRNAs or a nonspecific siRNA and subjected to Western blot analysis after $48 \mathrm{~h}$ of transfection for MyoD (upper panel) and $\alpha$-tubulin (lower panel) protein. The percent reduction in band intensity relative to nonspecific control is shown. (E) Schematic showing location of the shRNAs along the sequence of the MyoD gene. $(F)$ Normalized relative amount of EGFP and RFP for each shRNA. \% reduction in GFP fluorescence is indicated below each shRNA. (G) C2C12 cells were transfected with specific MyoD shRNAs or a nonspecific shRNA and subjected to Western blot analysis after $48 \mathrm{~h}$ of transfection for MyoD (upper panel) and $\alpha$-tubulin (lower panel) protein. shRNA against firefly luciferase and scrambled siRNA served as a nonspecific control. All of the blots were reprobed with anti- $\alpha$-tubulin (Sigma) antibody to show equal loading.

was included. Of the five siRNAs tested, siRNA 608 was by far the most effective in suppressing both ectopic Lamin-GFP fusion (60\% reduction in relative fluorescence intensity, Fig. 4B) and endogenous Lamin A/C (87\% reduction in lamin A/C protein level, Fig. 4C), in agreement with the $90 \%$ reduction observed previously using the same siRNA (Elbashir et al. 2001a). Similarly, in a screen of a panel of shRNA probes against the human tumor suppressor p53, a sequence known to be effective (Brummelkamp
637

897

MyoD

et al. 2002) performed most efficiently in our screen (Table 1). Taken collectively, these results validate the reliability of this approach for RNAi screening.

We have screened panels of siRNAs and shRNA probes against genes with diverse biological functions in both murine and human cell lines. Table 1 summarizes the screening results described above obtained with MyoD, Lamin A/C, and p53 as well as results obtained with the human EF-hand calcium binding protein $S 100 \alpha$-subunit and members of the Id family of genes. These results further underscore that only a minority of RNAi probes are effective in gene silencing, and that the effective RNAi probes can be identified from this screen. Thus far we have always observed a correlation between the efficiency of an siRNA in the screen and the suppression of the cognate endogenous gene. However, the generality of this correlation can only be determined by performing a comprehensive analysis involving a broad spectrum of genes. It is possible that factors such as enhanced transcriptional rates, protein stability, and density of RNA binding proteins associated with some genes may compromise the in vivo effectiveness of siRNA. This may result in a small proportion of false-negatives in this screening methodology.

Our assay can be used to screen for effective siRNAs directed against targets that cannot be monitored directly, either because of lack of reagents such as antibodies, or because of very low expression levels. Another strength of the screening method is its ability to identify the most robust siRNA candidate within $24 \mathrm{~h}$ of transfection irrespective of the status of the endogenous protein. This is particularly attractive compared to determining the efficacy of siRNA probes by directly monitoring their ability to suppress cognate endogenous genes, which may involve timeconsuming optimization of siRNA dose and incubation time (Elbashir et al. 2001a; Harborth et al. 2001; Mendez et al. 2002). In several cases we performed the screening using a variety of human and murine cell lines (HeLa, NIH3T3, murine endothelial, HEK293, IMR90, $\mathrm{C} 2 \mathrm{C} 12)$; in all cases the results were independent of the cell lines used (data not shown; also note that siRNA 25 and shRNA 708 against MyoD were identified using both murine C2C12 (Fig. 3F) and human HeLa cells [Fig. 5F,G]).

The screening method allows not only the identification of the most effective siRNAs, but also that of siRNAs that display partial suppression of target gene expression. We predict that such siRNAs would be useful in studies where partial downregulation of gene expression is desired, particularly in cases where partial downregulation might result in distinct phenotypes. For

\section{Genome Research}

www.genome.org 
A

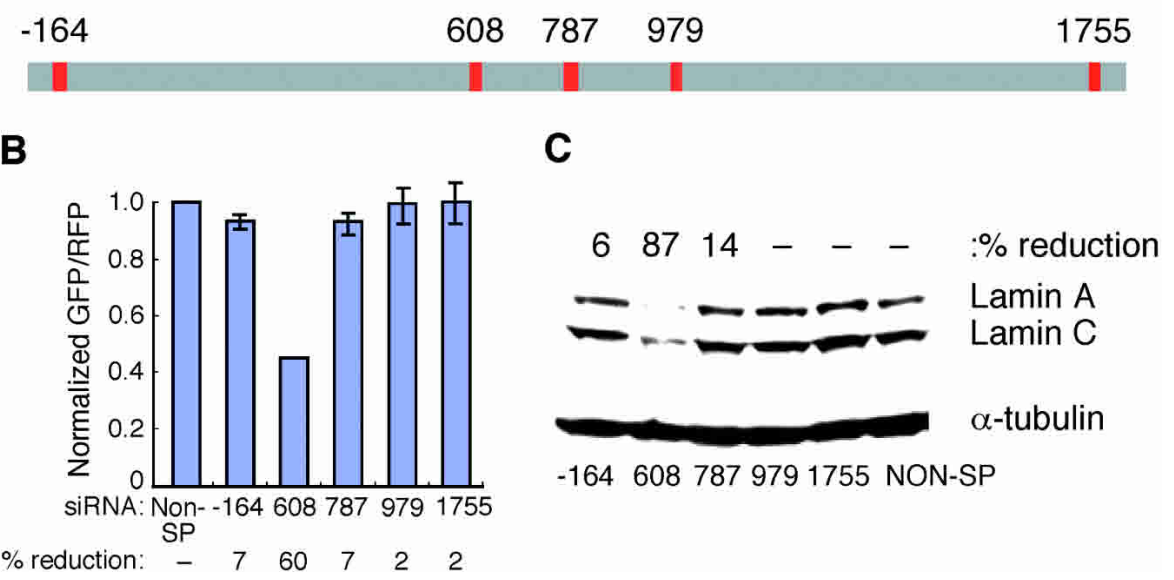

Figure 4 Correlation between siRNA screening results and suppression of endogenous Lamin A/C. $(A)$ The location of the siRNAs against the Lamin $A / C$ gene. $(B)$ HeLa cells were transfected with a constant amount of EGFP-Lamin A/C, pDsRed2-N1, and specific Lamin A/C siRNA and nonspecific siRNA. Normalized relative amounts of EGFP and RFP for each siRNA and \% reduction in GFP fluorescence for each siRNA are shown. (C) Western blot analysis using $\alpha$-Lamin A/C antibody.

example, shRNAs showing varying levels of p53 suppression generated distinct tumor phenotypes in vivo (Hemann et al. 2003). They would also be useful where lethality associated with complete suppression of critical genes is of concern.

\section{A High-Throughput Selection of siRNA Probes by RNAi Microarrays}

Currently a major effort is geared towards using RNAi as a reverse genetic approach for analyzing mammalian gene function on a genome-wide scale as demonstrated in C. elegans (Kamath et al. 2003). However unlike C. elegans, where long dsRNAs work well in sequence-specific gene silencing due to lack of an antiviral/ interferon response, successful applications using RNAi in somatic mammalian cells would require reliable RNAi screens to identify effective siRNA probes for every gene. We were interested in determining whether our method for selecting effective siRNA probes would work in a highly parallel assay. We made use of a DNA microarray platform where two plasmids, one encoding a target gene-EGFP fusion and another encoding RFP (as an internal control) were arrayed with either specific siRNA or nonspecific siRNA on a glass slide and then incubated with mammalian cell culture (Fig. 5A). Only the cells growing in close proximity to the DNA spots were transfected, resulting in spatially distinct groups of transfected cells within a lawn of untransfected cells, as observed previously (Ziauddin and Sabatini 2001). A laser scanner was used to monitor EGFP and RFP fluorescence intensities in each individual cluster. Figure 5C is a laser scan image of spots expressing EGFP (green) and RFP (red) expression, and Figure 5D is a microscopy image of a representative spot. Typically, each cluster was comprised of 300500 fluorescent cells. We next sought to determine whether the microarray-based cell transfection format would recapitulate siRNA-mediated suppression of ectopic gene expression as a function of siRNA concentration and specificity, as observed in conventional transfections (Fig. 2). Quantitation of normalized mean intensities (EGFP/RFP) showed a dose-dependent suppression of EGFP expression by its cognate siRNA (Fig. 5E), with 300 ng of siRNA providing maximal suppression. Moreover, an effective siRNA directed to EGFP showed specific suppression of EGFP and not RFP expression (Fig. 5B). All of these results are also consistent with those obtained in a conventional transfection assay (Fig. 2).

We then tested the use of cell microarrays to screen effective siRNA probes. First the concentrations of the target-reporter fusions were optimized for detection of fluorescence by a laser scanner (data not shown). Next, the panels of six siRNAs and six shRNAs directed against MyoD described above (Fig. 3A,E) were arrayed together with the plasmids encoding MyoD-EGFP and the internal control RFP. The quantitation of EGFP and the RFP internal control intensities in response to various shRNAs and siRNAs (Fig. 5F,G) identified shRNA 708 and siRNA 25 as the most effective in suppression of MyoD-EGFP expression, in agreement with our observations with conventional transfections (Fig. 3). Although by using conventional transfections we observed a partial suppression of MyoD gene expression by siRNA 294 (Fig. 3C,D) and shRNA 312 (Fig. 3G), the same probes did not show similar trends in the microarray-based screen (Fig. 5F,G). This is likely due to differences in the sensitivity and specificity of detection of the two screening platforms. Nevertheless, the two types of screening are in perfect agreement in their ability to identify the most effective RNAi probe.

These results suggest that the microarray-based cell transfections ("RNAi microarrays") can be used in large-scale RNAi screens. Using fully automated liquid-dispensing and platehandling robotic systems, it is possible to prepare mixtures containing constructs expressing target-reporter fusions, internal controls, various shRNAs, and synthetic siRNAs which then can be printed at densities of up to 6000 to 10,000 features per slide by modern microarrayers. Although similar screens can be carried out in 96- or 384-well plates, the microarray platform provides distinct advantages. The arrays are cost-effective because they require minimal materials (plasmids, shRNA, or siRNA) and expensive transfection reagents. Mass transfection on arrays allows homogenous distribution of cell population in comparison to variability often encountered in well-to-well transfections. Besides, several slides can be generated and the screens performed in duplicate or triplicate to obtain statistically significant results. However, a 96- or 384-well plate format is more suitable for enzymatic assays, such as for luciferase, $\beta$-galactosidase, etc.

Table 1. siRNA/shRNA-Induced Gene Silencing for Ectopically Expressed Target-Reporter Fusions

\begin{tabular}{llll}
\hline Gene & \multicolumn{1}{c}{ Accession } & siRNA $^{\text {a }}$ & shRNA $^{\text {a }}$ \\
\hline MyoD & M84918 & $5(1)$ & $5(1)$ \\
LaminA/C & NM_005572 & $5(1)$ & 0 \\
S-100 & NM_002961.2 & $5(0)$ & 0 \\
Id1 & AK008264 & $5(1)$ & $8(1)$ \\
Id2 & AF077860 & $5(0)$ & $3(1)$ \\
Id3 & AK002820 & $5(1)$ & $8(1)$ \\
Id4 & AF077859 & $5(1)$ & $3(0)$ \\
p53 & X02469 & $5(1)$ & $4(1)$ \\
\hline
\end{tabular}

a Of the total siRNA/shRNA tested in the screens, the number of the most effective probes are mentioned in parentheses. 


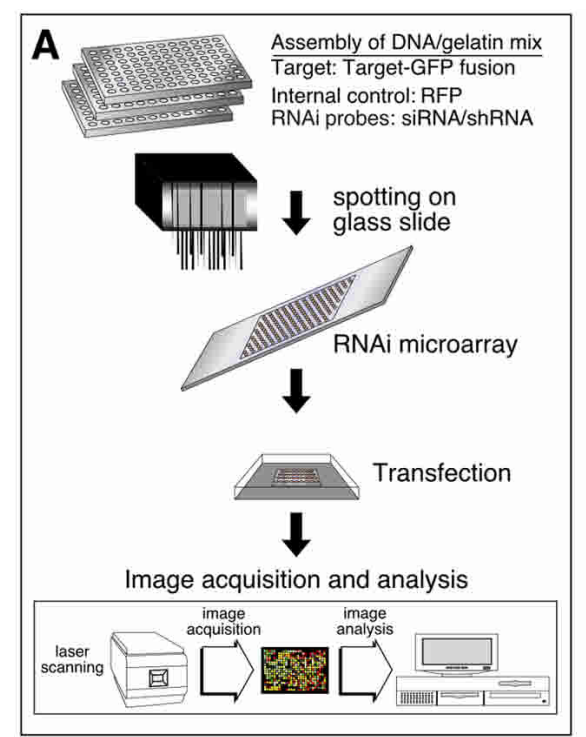

B

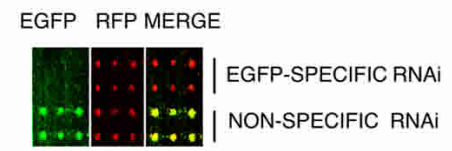

C

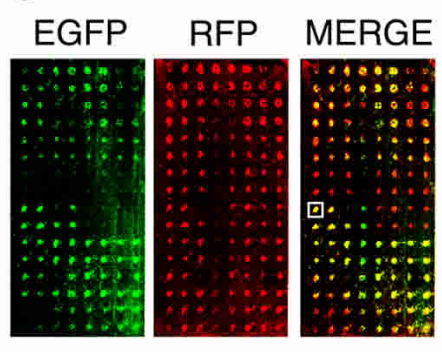

D

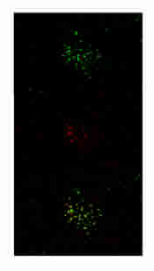

$\mathbf{E}$

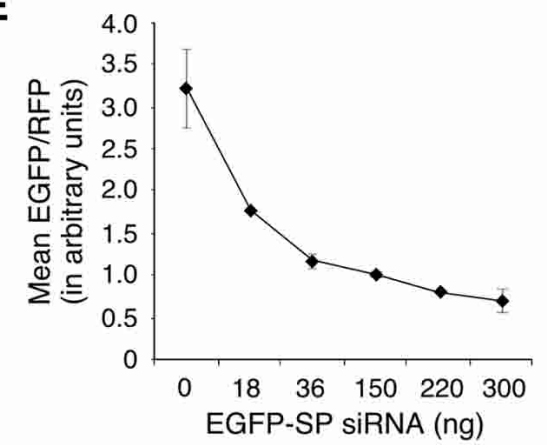

$\mathbf{F}$

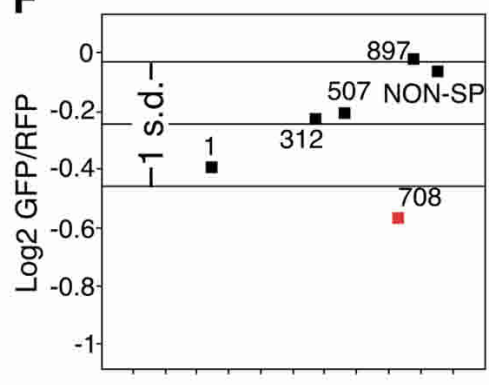

ShRNA

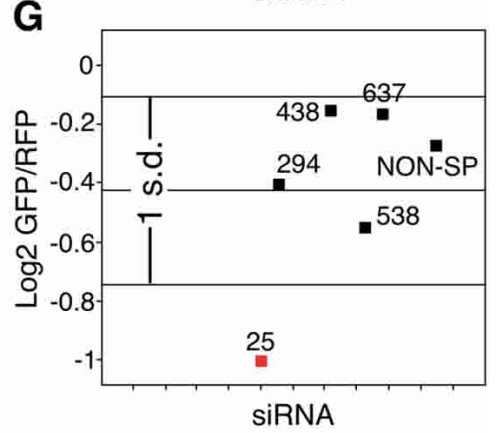

Figure 5 siRNA screens using microarray-based cell transfections. ( $A$ ) Schematic showing manufacture and analyses of an RNAi microarray. ( $B$ ) RNAi microarray showing that an effective EGFP specific siRNA results in the suppression of EGFP but not RFP expression. A nonspecific siRNA did not affect EGFP or RFP expression. (C) A laser scan image of EGFP- and RFP-expressing microarray. Each feature was $500 \mu \mathrm{M}$ in diameter with a pitch of $750 \mu \mathrm{M}$. $(D)$ Magnified image of a feature represented by a boxed area of an image from $A$. ( $E$ ). Dose-dependent response of EGFP-specific siRNA in suppression of EGFP expression as quantitated by mean trimmed normalized values of EGFP/RFP mean intensities $(n=4)$. $(F)$ Screen for identifying effective shRNA against $M y o D$ gene. $(G)$. Screen for identifying effective siRNA against MyoD. Normalized EGFP/RFP median intensities ( $y$-axis) were log transformed, normalized $(n=4)$, and plotted against individual siRNA/shRNA probes ( $x$-axis). In each case probes within and outside 1 standard deviation (s.d.) from the mean value were considered noneffective (marked in black) and effective (marked in red), respectively.

Although a major time constraint is the generation of constructs expressing the target-reporter fusion proteins, a novel cloning strategy to rapidly generate both $\mathrm{N}$ - and C-terminus GFP fusions in a high-throughput format is available (Simpson et al. 2000). This method allows for cloning of amplified open reading frames (ORFs) by recombination and therefore circumvents the need for screening for restriction sites in the ORF in question. Another possibility is the use of an internal ribosomal entry site (IRES) between the target and the reporter. This would bypass the stringent requirement of maintaining ORFs and simplify the cloning of target-reporter fusion constructs considerably. We observed weak IRES-mediated expression of GFP that was unsuitable for quantitation in our screening assays (data not shown).

We envisage that RNAi microarrays can also be directly exploited for scoring RNAi-generated phenotypes in mammalian cells that result from the loss of gene function, such as NuMa, GAS41, LaminA/C/B1/B2, $\beta / \gamma$ actin, zyxin, Eg5, CENP-E, and CdK1 shown previously in conventional transfection assays (Harborth et al. 2001).

In summary, we have designed a rapid, inexpensive, powerful, reliable, and quantitative method for screening the most effective siRNA or shRNA for suppressing gene expression in mammalian cells. This method is likely to be useful until detailed biochemical mechanisms of RNAi pathways are uncovered that will provide more rational strategies for efficiently targeting suppression of any desired gene. The ability to quickly identify effective siRNA for silencing any gene is likely to have significant implications not only in basic research, but also in RNAi-based therapeutics (Agami 2002; Cottrell and Doering 2003; Shi 2003) and in the generation of genetically modified animal models (Hasuwa et al. 2002; Kim et al. 2002; Carmell et al. 2003).

\section{METHODS}

\section{Generation of siRNAs, shRNAs, and Target-Reporter Fusion Constructs}

Synthetic siRNAs were either purchased from Dharmacon Research or synthesized by in vitro transcription (Donze and Picard 2002). Specific siRNA against EGFP (Donze and Picard 2002) and scrambled siRNA (sense 5'-ATGATACTCGAGGGCATGTCTCTATAGTGAGTCGTATTA-3' and antisense 5'-CGGAGACATGCCCTCGAGTATCTATAGTGAGTCGTATTA-3') were synthesized. siRNA and shRNA probes (see Supplemental Fig. 1, available online at www.genome.org) against target genes were designed using computer software (http://www.cshl.org/public/SCIENCE/ hannon.html). The location of the siRNAs and shRNAs on the target sequences were assigned with respect to the translational start site, ATG (where nucleotide A was designated position 1). shRNA sequences (two complementary 83-nt DNA oligos) were annealed and cloned directly into a U6 promoter-containing vector (Paddison et al. 2002) using a ligation-independent cloning method (S. Aruleba, K. Scobie, and D.S. Conklin, unpubl.). shRNA against firefly luciferase (Paddison et al. 2002) and a scrambled siRNA served as a nonspecific control. The MyoDEGFP fusion construct was prepared by amplifying MyoD cDNA (M 84918) from a pCMV-MyoDs vector and inserted into XhoI and BamHI sites of pEGFP-N2 (Clontech). EGFP-Renilla luciferase (EGFP-Rluc) fusion construct was prepared by cloning PCRamplified EGFP product from pEGFP-N2 (Clontech) into the

\section{Genome Research www.genome.org}


BamHI site of pRluc-N3 (PerkinElmer).

EGFP-Lamin A/C fusion construct was prepared by blunt end ligation (filled in by T4 DNA polymerase) of BsrGI and NotIdigested pEGFP-N2 vector with a NotI and SalI-digested Lamin A/C (NP_005563) insert from Lamin A/C-pSPORT I vector (Research Genetics). In all cases, the integrity and orientation of the target gene with respect to the reporter gene were confirmed by restriction enzyme digestion and DNA sequencing.

\section{Cell Culture, Transfection, and Western Blot Analysis}

Murine C2C12 and human HeLa cells (ATCC) were cultured in DMEM supplemented with $10 \% \mathrm{FBS}, 100 \mathrm{U} / \mathrm{mL}$ penicillin, and $100 \mu \mathrm{g} / \mathrm{mL}$ streptomycin (Life Technologies). Cells were transfected in 6-well plates using Lipofectamine PLUS (Life Technology) and $2 \mu \mathrm{g}$ of siRNAs or shRNAs. For Western blot analysis, cells were harvested at $48 \mathrm{~h}$ posttransfection and washed with TBS (50 mM Tris, pH 8.0, $150 \mathrm{mM} \mathrm{NaCl})$ and lysed in $100 \mu \mathrm{L}$ of RIPA lysis buffer (TBS supplemented with 1\% NP-40 and complete protease inhibitors, Roche Applied Science). Equal amounts of lysate were subjected to Western blot analysis (Harlow and Lane 1988) using $\alpha$-MyoD or Lamin A/C primary antibody (Santa Cruz) and horseradish peroxidase-conjugated secondary antibody. The blots were stripped twice by stripping buffer $(100 \mathrm{mM}$ $\beta$-mercaptoethanol, 2\% SDS, $62.5 \mathrm{mM}$ Tris- $\mathrm{HCl}, \mathrm{pH} 6.7)$ at $55^{\circ} \mathrm{C}$ and reprobed with anti- $\alpha$-tubulin (Sigma) primary antibody and horseradish peroxidase-conjugated secondary antibody. The blots were developed using an ECL Plus Western Blotting Detection System (Amersham Biosciences). Signal intensities were determined after background corrections by using Alpha-Imager 2000 Documentation and Analysis Software (Alpha Innotech). The percentage reduction in band intensity for each siRNA/ shRNA relative to nonspecific control (assumed to be unchanged) was calculated.

\section{Screening Assay}

For each well of a 24 -well plate, $150 \mathrm{ng}$ of siRNAs or shRNAs, 100 ng of target-reporter fusion construct, and $50 \mathrm{ng}$ of pdSRed2-N1 (internal control) were used in transfections. Twenty-four h posttransfection, EGFP and RFP images were captured using a Zeiss AxioCam HRm camera at equal exposure time for all panels. Cleared cell lysates were prepared as described above for the Western blot analysis, and EGFP and RFP intensities were quantitated using a Multilabel Counter (PerkinElmer) with Wallac 1420 software. The EGFP/RFP ratio was calculated and normalized against nonspecific control as described (Chiu and Rana 2002).

\section{Dual Luciferase Assay}

In each well of a 24-well plate, C2C12 cells were cotransfected with 300 ng EGFP-Rluc, 200 ng pGL3-Control (Promega), and increasing concentrations (12.5-250 ng) of specific EGFP siRNA or nonspecific siRNA. After $24 \mathrm{~h}$ of transfection, the relative amounts of Renilla and firefly luciferase were analyzed by a dual luciferase assay (Promega) using a luminometer (Model 3010, Analytical Scientific Instruments). The Renilla/firefly luciferase ratio was calculated and normalized against the control.

\section{Microarray-Based Cell Transfections (RNAi Microarray): Printing, Transfection, Laser Scanning, and Quantitation}

Essentially a mixture containing plasmid DNA and various siRNA or shRNA in $0.18 \%$ gelatin solution was prepared and arrayed in a 384-well plate. These were printed on CMT GAPS glass slides (Corning) by an arrayer (VP478A, V \& P Scientific) at $4^{\circ} \mathrm{C}$. The final plasmid concentrations for EGFP fusion construct or nucEGFP (Clontech) and pdSRed2-N1 (internal control) were 150 $\mathrm{ng} / \mu \mathrm{L}$ and $50 \mathrm{ng} / \mu \mathrm{L}$, respectively. The shRNA or siRNA concentration was kept constant at $300 \mathrm{ng} / \mu \mathrm{L}$ or as mentioned. For transfections, $24 \mu \mathrm{L}$ of Lipofectamine 2000 (Invitrogen) was mixed with $300 \mu \mathrm{L}$ of OPTI-MEM I media (GibcoBRL) and pipetted onto a $40 \times 20 \times 0.2 \mathrm{~mm}$ cover well (PC200; Grace Bio-
Labs). A slide with the printed side down was placed on the cover well and incubated for $45 \mathrm{~min}$. The cover well was removed and the slide was incubated with $1 \times 10^{6} \mathrm{HeLa}$ cells per $\mathrm{mL}$ for $24 \mathrm{~h}$ with a media change at $6 \mathrm{~h}$. The cells were fixed and mounted with a coverslip. More details on printing and transfection can be found at (Ziauddin and Sabatini 2001). The slides were scanned using a laser scanner (ScanArray 5000; PerkinElmer) at $20 \mu \mathrm{M}$ resolution to measure EGFP and RFP fluorescence. After scanning, the EGFP and RFP intensities of each spot were quantitated by GenePix 4.0 software (Axon Instruments). In all analyses, features showing obvious blemishes and morphological defects were eliminated. Features with low intensities ( $<100$ units) in the red channel (RFP fluorescence) were considered inefficient transfections and were removed from further analysis. Data were normalized by trimmed mean with the trim value set to $5 \%$ (highest $5 \%$ of the values and the lowest $5 \%$ of the values are excluded from the calculated mean) to reduce the effects of outliers. Each spot was represented in quadruplet, and average values were used for final quantitation. Data files are available as Supplemental Figures 2 and 3.

\section{ACKNOWLEDGMENTS}

We thank Nouria Hernandez, Eli Hatchwell, James Egan, and Sunita Gupta for comments on the manuscript and helpful discussions, and Greg Hannon, Jose Silva, Robert Benezra, Kimberly LaVine, Sunita Gupta, David Helfman, Eli Hatchwell, Sibel Kantaraci, and Andrew Lassar for reagents.

The publication costs of this article were defrayed in part by payment of page charges. This article must therefore be hereby marked "advertisement" in accordance with 18 USC section 1734 solely to indicate this fact.

\section{REFRENCES}

Agami, R. 2002. RNAi and related mechanisms and their potential use for therapy. Curr. Opin. Chem. Biol. 6: 829-834.

Bernstein, E., Caudy, A.A., Hammond, S.M., and Hannon, G.J. 2001. Role for a bidentate ribonuclease in the initiation step of RNA interference. Nature 409: 363-366.

Brummelkamp, T.R., Bernards, R., and Agami, R. 2002. A system for stable expression of short interfering RNAs in mammalian cells. Science 296: 550-553.

Bryom, M., Pallota, V., Brown, D., and Ford, L. 2002. Visualizing siRNA in mammalian cells: Fluorescence analysis of the RNAi effect. Ambion TechNotes 9: 3-5, www.ambion.com/tech/ib/tn/101/4.html.

Calegari, F., Haubensak, W., Yang, D., Huttner, W.B., and Buchholz, F. 2002. Tissue-specific RNA interference in postimplantation mouse embryos with endoribonuclease-prepared short interfering RNA. Proc. Natl. Acad. Sci. 99: 14236-14240.

Carmell, M.A., Zhang, L., Conklin, D.S., Hannon, G.J., and Rosenquist, T.A. 2003. Germline transmission of RNAi in mice. Nat. Struct. Biol. 10: $91-92$

Chiu, Y.L. and Rana, T.M. 2002. RNAi in human cells: Basic structural and functional features of small interfering RNA. Mol. Cell 10: $549-561$.

Cottrell, T.R. and Doering, T.L. 2003. Silence of the strands: RNA interference in eukaryotic pathogens. Trends Microbiol. 11: 37-43.

Donze, O. and Picard, D. 2002. RNA interference in mammalian cells using siRNAs synthesized with T7 RNA polymerase. Nucleic Acids Res. 30: 46 .

Elbashir, S.M., Harborth, J., Lendeckel, W., Yalcin, A., Weber, K., and Tuschl, T. 2001a. Duplexes of 21-nucleotide RNAs mediate RNA interference in cultured mammalian cells. Nature 411: 494-498.

Elbashir, S.M., Lendeckel, W., and Tuschl, T. 2001b. RNA interference is mediated by 21- and 22-nucleotide RNAs. Genes \& Dev. 15: 188-200.

Elbashir, S.M., Harborth, J., Weber, K., and Tuschl, T. 2002. Analysis of gene function in somatic mammalian cells using small interfering RNAs. Methods 26: 199-213.

Gil, J. and Esteban, M. 2000. Induction of apoptosis by the dsRNA-dependent protein kinase (PKR): Mechanism of action. Apoptosis 5: 107-114.

Hammond, S.M., Caudy, A.A., and Hannon, G.J. 2001. Posttranscriptional gene silencing by double-stranded RNA. Nat. Rev. Genet 2: 110-119.

Hannon, G.J. 2002. RNA interference. Nature 418: 244-251.

Harborth, J., Elbashir, S.M., Bechert, K., Tuschl, T., and Weber, K. 2001. Identification of essential genes in cultured mammalian cells using 


\section{Kumar et al.}

small interfering RNAs. J. Cell Sci. 114: 4557-4565.

Harlow, E. and Lane, D. 1988. Antibodies: A laboratory manual. Cold Spring Harbor Laboratory Press, Cold Spring Harbor, NY.

Hasuwa, H., Kaseda, K., Einarsdottir, T., and Okabe, M. 2002. Small interfering RNA and gene silencing in transgenic mice and rats. FEBS Lett. 532: 227-230.

Hemann, M.T., Fridman, J.S., Zilfou, J.T., Hernando, E., Paddison, P.J., Cordon-Cardo, C., Hannon, G.J., and Lowe, S.W. 2003. An epi-allelic series of p53 hypomorphs created by stable RNAi produces distinct tumor phenotypes in vivo. Nat. Genet. 3: 3 .

Hohjoh, H. 2002. RNA interference (RNA(i)) induction with various types of synthetic oligonucleotide duplexes in cultured human cells. FEBS Lett. 521: 195-199.

Holen, T., Amarzguioui, M., Wiiger, M.T., Babaie, E., and Prydz, H. 2002. Positional effects of short interfering RNAs targeting the human coagulation trigger tissue factor. Nucleic Acids Res. 30: 1757-1766.

Kamath, R.S., Fraser, A.G., Dong, Y., Poulin, G., Durbin, R., Gotta, M., Kanapin, A., Le Bot, N., Moreno, S., Sohrmann, M., et al. 2003. Systematic functional analysis of the Caenorhabditis elegans genome using RNAi. Nature 421: 231-237.

Kapadia, S.B., Brideau-Andersen, A., and Chisari, F.V. 2003. Interference of hepatitis $C$ virus RNA replication by short interfering RNAs. Proc. Natl. Acad. Sci. 100: 2014-2018.

Kim, M.H., Yuan, X., Okumura, S., and Ishikawa, F. 2002. Successful inactivation of endogenous Oct-3/4 and c-mos genes in mouse preimplantation embryos and oocytes using short interfering RNAs. Biochem. Biophys. Res. Commun. 296: 1372-1377.

Lee, N.S., Dohjima, T., Bauer, G., Li, H., Li, M.J., Ehsani, A., Salvaterra, P., and Rossi, J. 2002. Expression of small interfering RNAs targeted against HIV-1 rev transcripts in human cells. Nat. Biotechnol. 20: $500-505$

Marcus, P.I. and Sekellick, M.J. 1985. Interferon induction by viruses. XIII. Detection and assay of interferon induction-suppressing particles. Virology 142: 411-415.

McManus, M.T., Petersen, C.P., Haines, B.B., Chen, J., and Sharp, P.A. 2002. Gene silencing using micro-RNA designed hairpins. $R N A$ 8: $842-850$.
Mendez, J., Zou-Yang, X.H., Kim, S.Y., Hidaka, M., Tansey, W.P., and Stillman, B. 2002. Human origin recognition complex large subunit is degraded by ubiquitin-mediated proteolysis after initiation of DNA replication. Mol. Cell 9: 481-491.

Paddison, P.J., Caudy, A.A., Bernstein, E., Hannon, G.J., and Conklin, D.S. 2002. Short hairpin RNAs (shRNAs) induce sequence-specific silencing in mammalian cells. Genes \& Dev. 16: 948-958.

Paul, C.P., Good, P.D., Winer, I., and Engelke, D.R. 2002. Effective expression of small interfering RNA in human cells. Nat. Biotechnol. 20: $505-508$.

Sharp, P.A. 1999. RNAi and double-strand RNA. Genes \& Dev. 13: $139-141$.

Shi, Y. 2003. Mammalian RNAi for the masses. Trends Genet. 19: 9-12.

Simpson, J.C., Wellenreuther, R., Poustka, A., Pepperkok, R., and Wiemann, S. 2000. Systematic subcellular localization of novel proteins identified by large-scale cDNA sequencing. EMBO Rep. 1: 287-292.

Sui, G., Soohoo, C., Affar el, B., Gay, F., Shi, Y., and Forrester, W.C. 2002. A DNA vector-based RNAi technology to suppress gene expression in mammalian cells. Proc. Natl. Acad. Sci. 99: 5515-5520.

Yang, D., Buchholz, F., Huang, Z., Goga, A., Chen, C.Y., Brodsky, F.M. and Bishop, J.M. 2002. Short RNA duplexes produced by hydrolysis with Escherichia coli RNase III mediate effective RNA interference in mammalian cells. Proc. Natl. Acad. Sci. 99: 9942-9947.

Yu, J.Y., DeRuiter, S.L., and Turner, D.L. 2002. RNA interference by expression of short-interfering RNAs and hairpin RNAs in mammalian cells. Proc. Natl. Acad. Sci. 99: 6047-6052.

Ziauddin, J. and Sabatini, D.M. 2001. Microarrays of cells expressing defined cDNAs. Nature 411: 107-110.

\section{WEB SITE REFERENCES}

http://www.cshl.org/public/SCIENCE/hannon.html; RNAi OligoRetriever.

Received May 22, 2003; accepted in revised form August 1, 2003. 


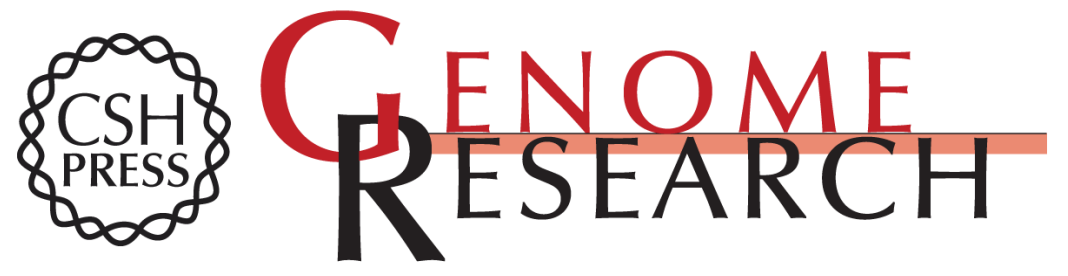

\section{High-Throughput Selection of Effective RNAi Probes for Gene Silencing}

Rajeev Kumar, Douglas S. Conklin and Vivek Mittal

Genome Res. 2003 13: 2333-2340

Access the most recent version at doi:10.1101/gr.1575003

Supplemental http://genome.cshlp.org/content/suppl/2003/09/10/13.10.2333.DC1

Material

References This article cites 32 articles, 10 of which can be accessed free at: http://genome.cshlp.org/content/13/10/2333.full.html\#ref-list-1

\section{License}

Email Alerting Receive free email alerts when new articles cite this article - sign up in the box at the Service top right corner of the article or click here.

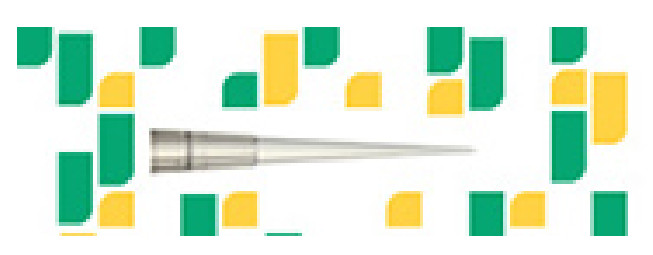

To subscribe to Genome Research go to: https://genome.cshlp.org/subscriptions 